\title{
A STABILITY PROPERTY OF A CLASS OF BANACH SPACES NOT CONTAINING A COMPLEMENTED COPY OF $l_{1}$
}

\author{
ELIAS SAAB AND PAULETTE SAAB
}

\begin{abstract}
Let $E$ be a Banach space and $K$ be a compact Hausdorff space. The space $C(K, E)$ will stand for the Banach space of all continuous $E$-valued functions on $K$ equipped with the sup norm. It is shown that the space $E$ does not contain a complemented subspace isomorphic to $l_{1}$ if and only if $C(K, E)$ has the same property.
\end{abstract}

Let $E$ be a Banach space, let $(\Omega, \Sigma, \lambda)$ be a finite measure space. The classical Banach spaces $l_{1}, c_{0}, L_{p}(\lambda)$ and $L_{p}(\lambda, E)$ will have their usual meaning [3]. The notations and terminology used and not defined in this paper can be found in [3], [4], [7].

In [5] Kwapien showed that if $c_{0}$ embeds in $L_{p}(\lambda, E)$ then $c_{0}$ embeds into $E$ if $1<p<+\infty$. Pisier [6] showed that if $l_{1}$ embeds into $L_{p}(\lambda, E)$ then $l_{1}$ embeds in $E$ if $1<p<+\infty$. The moral behind Kwapien's theorem is: Since $c_{0}$ cannot embed into $L_{p}(\lambda)$ if $1 \leqslant p<+\infty, c_{0}$ must embed into $E$ if it does embed in $L_{p}(\lambda, E)$. A similar remark can be made about Pisier's result.

In this paper we will show that if $l_{1}$ is isomorphic to a complemented subspace of $C(K, E)$, then $l_{1}$ is isomorphic to a complemented subspace of $E$ and the moral behind our result is that $l_{1}$ is not isomorphic to a complemented subspace of any $C(K)$ space.

THEOREM 1. Let $K$ be a compact Hausdorff space and $E$ be a Banach space; then $l_{1}$ is isomorphic to a complemented subspace of $C(K, E)$ if and only if $l_{1}$ is isomorphic to a complemented subspace of $E$.

Proof. If $l_{1}$ is isomorphic to a complemented subspace of $C(K, E)$, then $c_{0}$ embeds in $C(K, E)^{*}[1]$. The space $C(K, E)^{*}$ is isometrically isomorphic to the Banach space $M\left(K, E^{*}\right)$ of all $w^{*}$-regular $E^{*}$-valued measures of bounded variation defined on the $\sigma$-field $\Sigma$ of Borel subsets of $K$ and equipped with the norm $\|m\|=|m|(K)$, where $|m|$ is the variation of $m$. Let $\left(m_{n}\right)_{n>1}$ be a sequence in $M\left(K, E^{*}\right)$ equivalent to the usual $c_{0}$-basis and let $\lambda$ be the scalar measure defined on $\Sigma$ by $\lambda=\sum_{n=1}^{\infty}\left|m_{n}\right| / 2^{n}$. Let $\Sigma_{1}$ be the completion of $\Sigma$ with respect to $\lambda$.

Received by the editors December 20, 1980.

1980 Mathematics Subject Classification. Primary 46G10, 46B22.

Key words and phrases. Complemented subspaces, vector measures.

(c) 1982 American Mathematical Society 0002-9939/82/0000-0010/\$01.75 
Fix $\rho$ a lifting of $\mathfrak{L}^{\infty}\left(\Sigma_{1}, \lambda\right)[4, \S 11]$. For each $n>1$, there exists a function $g_{n}$ : $K \rightarrow E^{*}$ such that

(i) For every $x \in E$, the map $t \rightarrow\left\langle g_{n}(t), x\right\rangle$ is $\lambda$-integrable.

(ii) For every $A \in \Sigma_{1}$ and every $x \in E$

$$
\left\langle m_{n}(A), x\right\rangle=\int_{A}\left\langle g_{n}(t), x\right\rangle d \lambda .
$$

(iii) $\rho\left(g_{n}\right)=g_{n}$ (see $[4$, p. 212]).

(iv) The map $t \rightarrow\left\|g_{n}(t)\right\|$ is $\lambda$-integrable and $\left\|m_{n}\right\|=\int_{K}\left\|g_{n}(t)\right\| d \lambda$.

The existence of each $g_{n}$ satisfying (i)-(iv) is assured by [4, $\$ 11$, Theorem 5]. Let $\left(a_{n}\right)_{1<n<p}$ be a finite real sequence and let $m=\sum_{n=1}^{p} a_{n} m_{n}$. By [4, $\$ 11$, Theorem 5], there exists a function $g: K \rightarrow E^{*}$ satisfying with respect to $m$ and $\lambda$ the above properties (i)-(iv); therefore $\rho(g)=g$ and $\|m\|=\int_{K}\|g(t)\| d \lambda$, and for every $x \in E$ and every $A$ in $\Sigma_{1}$

$$
\langle m(A), x\rangle=\int_{A}\langle g(t), x\rangle d \lambda .
$$

Let $h=\sum_{n=1}^{p} a_{n} g_{n}$; the properties (ii) and (*) imply that for every $x \in E$

$$
\langle h(t), x\rangle=\langle g(t), x\rangle, \lambda \text {-almost everywhere. }
$$

The properties (**), $\rho(g)=g$ and $\rho(h)=h$ imply that $g=h[4$, p. 212]. Hence

$$
\left\|\sum_{n=1}^{p} a_{n} m_{n}\right\|=\int_{K}\left\|\sum a_{n} g_{n}(t)\right\| d \lambda
$$

for any finite sequence of reals $\left(a_{n}\right)_{1<n<p}$. Let $F$ be the space of all finite real sequences and denote by $e_{n}$ the $n$th unit vector. For $a=\left(a_{n}\right)_{n} \in F$, let $\|a\|_{\infty}=$ $\sup _{n}\left|a_{n}\right|$. For each $t \in K$ define a seminorm ||$_{t}$ on $F$ by $|a|_{t}=\left\|\Sigma a_{n} g_{n}(t)\right\|$. Clearly $|a|=\int_{K}|a|_{t} d \lambda$ is a seminorm of $F$ and $|a|=\left\|\Sigma a_{n} m_{n}\right\|$. Since $\left(m_{n}\right)$ is equivalent to the $c_{0}$-basis in $M\left(K, E^{*}\right)$ we have

$$
C_{2}\|a\|_{\infty} \leqslant\left\|\sum a_{n} m_{n}\right\| \leqslant C_{1}\|a\|_{\infty}
$$

for some $C_{1}>0$ and some $C_{2}>0$, but this implies that $C_{2}\|a\|_{\infty} \leqslant|a| \leqslant C_{1}\|a\|_{\infty}$ and this means that $\left(e_{n}\right)_{n>1}$ is equivalent to the $c_{0}$ basis for $\|$ in $F$. By [2, Theorem 1], there exist $t \in K$ and a subsequence of $\left(e_{n}\right)_{n>1}$ which is a $c_{0}$-basis for ||$_{t}$. Hence there exists a subsequence $\left(g_{n_{p}}\right)$ of $\left(g_{n}\right)$ such that $\left(g_{n_{p}}(t)\right)$ is equivalent to the usual $c_{0}$-basis in $E^{*}$; therefore $c_{0}$ embeds in $E^{*}$. Consequently $l_{1}$ is isomorphic to a complemented subspace of $E$ by [1]. The other implication is of course obvious.

Suppose now that $K$ is a compact convex subset of a locally convex Hausdorff topological vector space and let $A(K, E)$ stand for the Banach space of all affine $E$-valued continuous functions equipped with the supremum norm. Theorem 1 and Theorem 3.4 of [7] give a more general result, namely,

Corollary 2. Suppose that $K$ is a Choquet simplex. Then $l_{1}$ is isomorphic to a complemented subspace of $A(K, E)$ if and only if $l_{1}$ is isomorphic to a complemented subspace of $E$. 
Proof. Note that since $K$ is a Choquet simplex, the dual of $A(K, E)$ is isometrically isomorphic to a closed subspace of $M\left(K, E^{*}\right)$ [7, Theorem 3.4]. By [1], $c_{0}$ embeds in $A(K, E)^{*}$ and therefore $c_{0}$ embeds in $M\left(K, E^{*}\right)$. Hence $c_{0}$ embeds in $E^{*}$ by Theorem 1 and consequently $l_{1}$ is isomorphic to a complemented subspace of $E$.

\section{BIBLIOGRAPHY}

1. C. Bessaga and A. Pelczynski, On bases and unconditional convergence of series in Banach spaces, Studia Math. 17 (1958), 151-164.

2. J. Bourgain, An averaging result for $c_{0}$-sequences, Bull. Soc. Math. Belg. Ser. B 30 (1978), 83-87.

3. J. Diestel and J. J. Uhl, Vector measures, Math. Surveys, no. 15, Amer. Math. Soc., Providence, R.I., 1977.

4. N. Dinculeanu, Vector measures, Pergamon Press, New York, 1967.

5. S. Kwapien, Sur les espaces de Banach contenant $c_{0}$, Studia Math. 52 (1974), 187-188.

6. G. Pisier, Une propriété de stabilité de la classe des espaces ne contenant pas $l_{1}, \mathrm{C}$. R. Acad. Sci. Paris Sér. A 286 (1978), 747-749.

7. P. Saab, The Choquet integral representation in the affine vector valued case, Aequationes Math. 20 (1980), 252-262.

Department of Mathematics, University of Brttish Columbia, Vancouver, Brtish Columbia, CANADA

Current address: Department of Mathematics, University of Missouri-Columbia, Columbia, Missouri 65211 